\title{
Probability Based Search Motion Estimation Algorithm Using Mean Correction
}

\author{
Deepak J. Jayaswal ${ }^{1}$ and Mukesh A. Zaveri ${ }^{2}$
}

\begin{abstract}
We propose a stochastic approach to determine the motion vector (MV) for block matching algorithm (BMA). This approach allows us to exploit random distribution of motion vector in successive video frames from which the initial candidate predictors are derived. The derived predictors are the most probable points in search window, which will assure that, the motion vectors in the vicinity of center point and at the edge of the search window does not miss out, as it does for earlier algorithms like Three step search(TSS), Four step search(FSS), Diamond(DS), etc and refinement stage used in the algorithm will allow us to extract true motion vector so that the picture quality is as good as Full $\operatorname{search}(\mathrm{FS})$ which is the optimal algorithm. The novelty of the proposed algorithm is that the search pattern derived is not static but can dynamically shrink or enlarge to account for small and large motion. It is important to note that for the first time mean correction technique is introduced in video codec to improve PSNR with early termination of the algorithm. The Simulation result shows that our proposed algorithm outperforms all sub-optimal algorithms in terms of quality and speed up performance and in many cases PSNR of proposed algorithm is comparable or better than Full Search.
\end{abstract}

Index Terms - Motion vector, Block matching algorithm, Three step search, Four step search, Diamond search, Full search

\section{INTRODUCTION}

Motion estimation (ME) and compensation are the keys to high quality video coding [1]. ME is a process of estimating the motion of macroblock (MB) of some predefined size from the reference frame to generate the current frame. Block Matching based motion estimation is used in most video codecs, including MPEG-2, MPEG4 and H.263 [2]. ME is also a key component in the digital restoration of achieved video and for post production and special effect in the movie industry. ME and the exploitation of the strong correlation among the successive frames allows us to encode and transmit the motion vectors along with the error frame obtained using the regenerated current frame and the actual current frame and hence, reduces the number of bits used to convey the information. To achieve this bit reduction, various approaches and algorithms have been proposed in the literature [1] [2] [ 3] [24] [27]. The most accurate BMA is the exhaustive FS method, which exhaustively evaluates all

Manuscript received September 2, 2009.

D. J. Jayaswal is with the St Francis Institute of Technology, Mumbai, India. (Phone: +91-22-28928585; fax: +91-22-28954787; e-mail: djjayaswal_vcet@yahoo.com).

M. A. Zaveri is with the Sardar Vallabhai National Institute of Technology, Surat, India (e-mail: mazeveri@coed.svnit.ac.in ). possible macro blocks ( $\mathrm{p} \times \mathrm{p}$ ) over a predetermined search window of size $(2 p+1) \times(2 p+1)$ to find the best match. The estimated motion vector is the best match achieved for a predefined block distortion measure (BDM). The only disadvantage of this method and perhaps the biggest flaw is the high computational cost associated with it. Other algorithms with reduced number of computations, for example, the successive elimination algorithm (SEA) [4], a new three step search [5], a novel four-step search (FSS) [6], efficient four step search [7], unrestricted center biased diamond search (UCBDS) [8], cross search [9], fast full search motion estimation [10], complexity bounded motion estimation [11], new fast algorithm for estimation of block motion vector [12], dynamic search window algorithm [13], predictive coding based on interframe efficient motion estimation [14], displacement measurement and its application in image coding [15], a new efficient block-matching algorithm for motion estimation [16], fast variable block-size motion estimation algorithms based on merge and split procedure [17], Among these algorithms, the SEA is similar to the full search method except, the first one eliminates certain search points based on the Minkowiski's inequality. Further reduction in number of search points was achieved in TSS algorithm which starts with a step having nine uniformly spaced search points which get closer after every step until the step size reduces to 1 . The best candidate search point in the previous step becomes the center of the current step. The main drawback of TSS is the relatively large search pattern in the first step having a distance of 4 , which renders it inefficient for finding blocks with small motions. In order to exploit the characteristics of the center-biased motion vector distribution, FSS algorithm was proposed to speed up the search mechanism. It utilizes a nine-point search pattern on a 5 X 5 grid in the first step. FSS requires only four search steps as it starts with a smaller search grid pattern. The total number of candidate search points in FSS actually ranges from 17 (the best case) to 27 points (the worst case). The main drawback of FSS is the relatively small search pattern in the first step having a distance of 2, which renders it inefficient for finding blocks with large motions. TSS, FSS algorithm are static that is they have fixed search pattern and restricted window size, and are inefficient for predicting true motion vector for low or high motion. Other algorithm like appeared in [18-27] uses motion vector prediction (MVP) technique and has shown significant performance improvement in terms of quality. These algorithms provide better peak to signal ratio (PSNR) as compared to TSS, FSS, DS, etc. and are not static but are very complex and needs memory to store motion vectors for 
prediction. This makes it difficult for multimedia application where porting of video codec for embedded processor is required as well as for video streaming application for video on demand. Almost all of the BMAs make explicit and implicit assumptions that the matching distortion increase monotonically as the checking point moves away from the global minimum or the error surface is unimodal over the global window. Indeed, this assumption is not always true. Consequently, the resultant MVs may be trapped in a local minimum. Most BMAs exhibit proper behavior provided the following prerequisites are met [17]: 1) object displacement is constant within a block of pixels; 2) pixel illumination between successive frame is spatially and temporally uniform (this constraint can be relaxed in the BMA with luminance correction); 3) motion is restricted to translation; 4) matching distortion increases monotonically as the displaced candidate block moves away from the direction of the exact minimum distortion. Most of these conditions are usually not met for real-life video sequences, but a large number of $\mathrm{ME}$ algorithms still perform reasonably well. There are two major disadvantages of these algorithms (i) these algorithms yield different performance on different video sequences and (ii) their high complexity. In this paper we suggest a stochastic approach to determine the initial candidate predictor that is the most probable search point for first iteration and it is followed by refinement stage which allow us to extract true motion vector so that picture quality is as good as FS. The designed search pattern as mentioned earlier can dynamically shrink or enlarge depending on mean and threshold criteria to account for small and large motion. The algorithm proposed has a the search pattern which is derived from the knowledge of probability distribution of motion vector in given search window and concept of mean correction, will ensures that, 1) the search is not trapped in the local minima, 2) the search does not miss out on the motion vector found in the central region, 3) the search is terminated early by using mean correction and threshold without sacrificing PSNR, 4) PSNR is improved using mean correction technique, 5) computations are less 6) complexity is low, 7) memory is not needed to store motion vectors as it is required in most predictive ME algorithm. This paper proposes a PBSMC algorithm and the organization of the paper is as follows: Section II presents the review of motion estimation algorithm. Section III presents performance evaluation measurements. Section IV presents proposed algorithm and explains the algorithm in detail considering the steps involved. Section V presents some simulation results of our proposed algorithm in comparison with the algorithms like the FS, SEA, TSS, 4SS and DS.

\section{REVIEW Of Motion Estimation Algorithm}

Suboptimal motion estimation algorithm develop in recent years can be broadly classified as follows.

1) Heuristic Search Techniques: Instead of searching all candidates within search area, it looks for less number of candidate MVs in search area. The choice of the position is driven by some heuristic criterion in order to find the absolute minimum of cost function. The most famous variant of this technique are TSS, NTSS, FSS, and DS.

2) Fast Exhaustive Search Technique: In these technique reduction of search points is obtained by removing many of the nonoptimal search position without losing the optimality of the FS algorithm [SEA].

3) Hierarchical or Multiresolution Technique: The MVs are searched for a low resolution image and the refined in the normal resolution.

4) Spatio-Temporal Correlation Technique: The MVs are selected using the vectors that have already been calculated in the current and in the previous frames. Often this vector is starting point of a refinement stage

The algorithm presented in this paper belongs to heuristic and spatio-temporal technique category: the algorithm in facts finds the best initial predictor MV between a restricted set of vectors and refines it in a successive phase. Due to the presence of initial predictor which is decided on knowledge of MV distribution in given search window and its ability to switch between search patterns and improve quality by mean correction the algorithm is named as probability based search motion estimation with mean correction(PBSMC).

\section{PERFormanCE EVALUATION MEASUREMENT}

There are several performance measure function to test the efficiency of a MV, each of which weighs the pointto-point difference between two MBs: the PBSMC uses the SSE function (sum of square error) as it offers a good evaluation precision.

$$
\operatorname{SSE}(m, n)=\sum_{i, j}\left[B(i, j)-B(i+m, j+n 0]^{2}\right.
$$

with i,j $€$ macroblock

where $B(i, j)$ is the pixel value in the $(i, j)$ position of the current frame and $B^{\prime}(i+m, j+n)$ is the corresponding pixel value of the previous frame, in a position that has been shifted by a MV of the $(m, n)$ components.

\section{PRoposed PBSMC AlgorithM}

Our algorithm is based on stochastic approach which exploit random distribution of MVs in successive video frames for selection of search points within given search window, with the assumption that motion field varies slowly both spatially and temporally, therefore it is highly probable that $\mathrm{MBs}$ closer to the current $\mathrm{MB}$, in time and space may show the same motion. If this assumption holds, instead of testing all possible MVs, as FS does, we can use the most probable MV that is the set of candidate predictors. Then the best candidate predictor, that is the MV with the lowest SSE, is sent to a refinement phase that allows obtaining the final MV. The algorithm described here operates in two phases. In first phase we determine the initial candidate predictor to begin the search and also ensure that search does not get trap in local minima and in second phase the refinement stage will look for true motion vector.

\section{A. Initial candidate predictor:}

The driving idea is to reduce the cardinality of the candidate predictor set as much as possible and, at the same time, to put the "best" candidate in the set that is those MV that describe at best the motion. In order to find the best 
candidate for initial search,

We propose the basic search pattern as shown in Fig. 1. The search points in the central diamond region, at location $(0,0)$ $(0,-1)(0,-2)(0,1)(0,2)(-1,-1)(-1,0)(-1,1)(-2,0)(1,1)(1,0)$ $(1,1)(2,0)$ are 13 most probable points which are derived by determining the motion vector distribution probability over a search window of $(2 p+1) \times(2 p+1)$.

- central diamon d REgION

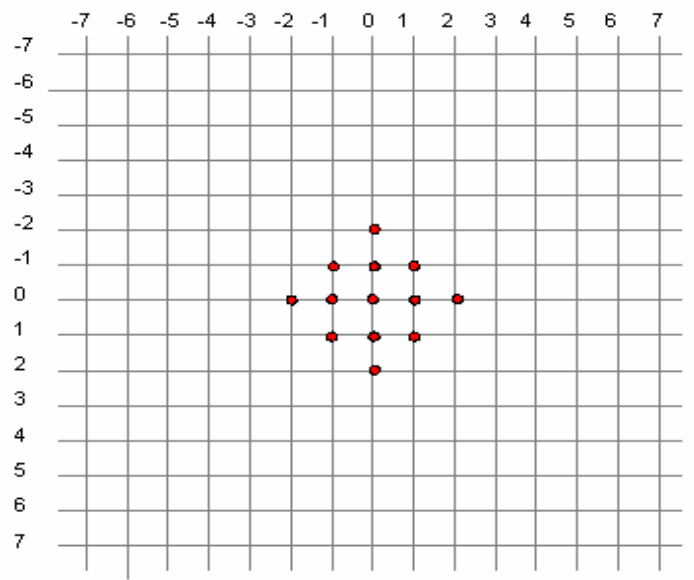

Fig.1. Basic search pattern of PBSMC

Table I depicts the percentage motion vector distribution probability over search window of $15 \times 15(\mathrm{p}=7)$ for multiple frames of QCIF sequence using full search (FS) motion estimation algorithm. FS finds motion vectors by sequentially searching the whole $15 \times 15$ search window in the reference frame. A MB centered at each of the position in the window is compared to the MB in the target frame, pixel by pixel, and their respective sum of square (SSE) is then derived. The vector $(i, j)$ that offers the least MAD is designated the motion vector $\mathrm{MV}(\mathrm{u}, \mathrm{v})$ for the $\mathrm{MB}$ in the target frame. For each of the MV detected at location $(i, j)$ in $15 \times 15$ search window a count is maintained for multiple frames of the sequence. The percentage MVP at a particular location $(\mathrm{i}, \mathrm{j})$ is

$\% \operatorname{MVP}(\mathbf{i}, \mathbf{j})=100 *$ (Number of MVs found at location(i,j) / Total number of MVs)

$=100 *($ Count $(\mathbf{i}, \mathbf{j}) /$ Total number of MVs $)$

For example if we consider 75 frames with frame size of $320 \times 240$ and $\mathrm{MB}$ size of $8 \times 8$, we have total $40 \times 30 \times 75$ numbers of MVs. If count at $(i, j)$ location using FS equals

15 then $\% \mathbf{M V P}(\mathbf{i}, \mathbf{j})=\mathbf{1 5 x} \mathbf{1 0 0}=\mathbf{0 . 1 1 \%}$

As observed from Table.1, the motion vector distribution is highly center biased with about $75 \%$ of the motion vectors being found in the central diamond region. To further justify our selection of 13 points in central diamond region, we analyze the shortcoming in earlier suboptimal algorithm like TSS, FSS, and DS. It is well known that the PSNR of these algorithms are low as compared to FS, the cause of failure of these algorithms is initial static search pattern which is unable to detect true motion vector that is TSS, FSS, and DS fails to find best match that FS has been able to find at particular location within $15 \times 15$ search window. To analyze the pitfalls of these algorithms we have generated error table using varieties of video sequences but we have shown result of akiyo sequence in Table. II - IV where each entry in table indicates the percentage error that is number of times TSS, FSS, and DS fail to find the true motion vector for multiple frames that FS has been able to find at particular location within $15 \times 15$ search window. The percentage error at location

$(i, j)$ is

$\%$ error $(i, j)=100 *\{1-[$ [number of MVs found by TSS at (i,j) / number of MVs found by FS at (i,j)] \}

The zero percent error at nine location in Table II-IV is reflection of initial search points of TSS, FSS, and DS respectively but error accumulation in inner diamond region at location $(-1,0)(0,-1)(1,0)(0,1)$ is about $45 \%$ for TSS, $35 \%$ for FSS and $24 \%$ for DS, clearly diamond search performs better than other two algorithm but still fails to account for MVs in inner diamond region. Therefore it is mandatory for any motion estimation algorithm, not to miss out on any MVs found in central diamond region.

Based on the probability distribution of motion vector and error table, our next logical step was to decide on number of search points in proximity of central point that is $\operatorname{mv}(0,0)$, for this we selected maximum 13 most probable points in central diamond region. We found that this 13 most probable points as shown in Fig.1. gives better PSNR result than diamond search at cost of few additional points. However, from simulation result of Table VIII-IX, it is observed, this algorithm, just like diamond search, gets trapped in local minima and often fails to find motion vectors which are closer to edge of the search window. In order to solve the problem of local minima trap and also look for the motion vector located at edge of search window. We propose the search pattern of Fig. 2 as initial search for first iteration with most probable points in central diamond region and next probable 8 points in outer diamond region located at $(6,0)$ $(-3,-3)(-3,3)(0,-6)(0,6)(3,-3)(3,3)(6,0)$ to take care of motion vector located at edge of search window.

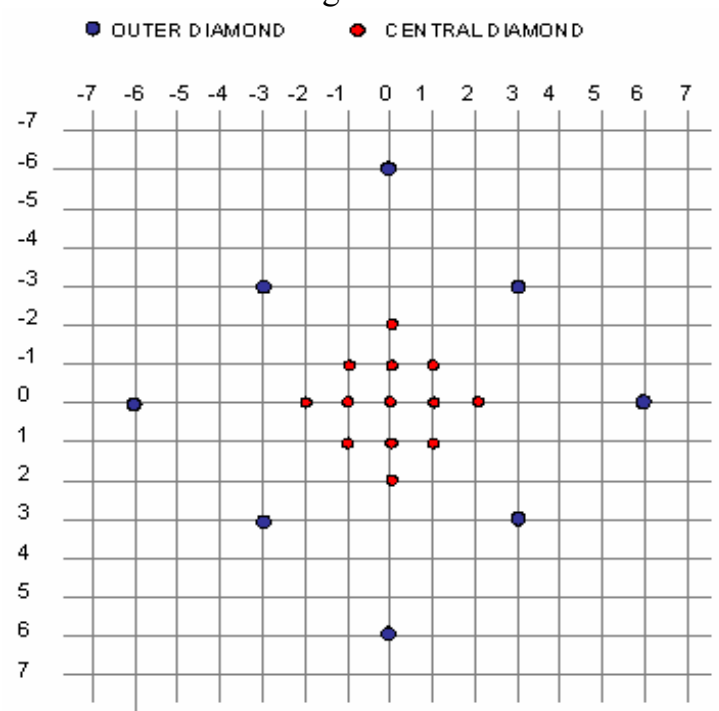

Fig.2. Search pattern of PBSMC (PBSMCT)

This pattern has ability to shrink or enlarge depending on criteria mentioned, the shrinking can be as small as five points and enlarge may be as big as twenty one points. After initial iteration, if minimum cost function is found in the central diamond region the refinement stage uses eight point search (EPS) described in section IV-B and if minimum cost 
function is found in outer diamond the search algorithm uses DS to track true motion vector. Simulation result will show that this pattern do not get trap in local minima as well as look for true motion vector.

\section{B. Refinement Stage:}

In this phase the refinement vectors try to correct the best candidate predictor searched in first phase to achieve the true motion vector. The refinement is done on the vectors obtain in first iteration to ensure that algorithm does not get trapped in local minima. Refinement phase let the motion field evolve and provide the required resolution. Since there is not a preferred direction, the refinement vectors are placed as a grid, centered on the best predictor, made of four points on cross direction and four on diagonal directions for motion vector found in central diamond region. These correction vectors of the refinement grid have a very limited range, one pixel, but they are able to give a good correction in most cases. However, in some circumstances, for example the MV found at the edge of the search window, it is convenient to enlarge the grid to recover the correct motion, in other words use diamond search to locate true motion vector.

In our algorithm refinement grid shrinks if minimum SSE is found in central diamond that is EPS used. It enlarges if minimum SSE is found in outer diamond that is DS algorithm used. We consider the eight neighbors around the central point. If a match is found at the center, the search is terminated. If a match is found at any of the neighbors, it is considered as the central point for the next iteration in the search. The search then continues in this manner till a central point is found as the match.

- Eight point search (EPS)

The basic search-point configuration of the EPS is as shown in the Fig. 3

- CEnTRAL DIAMOND REgION

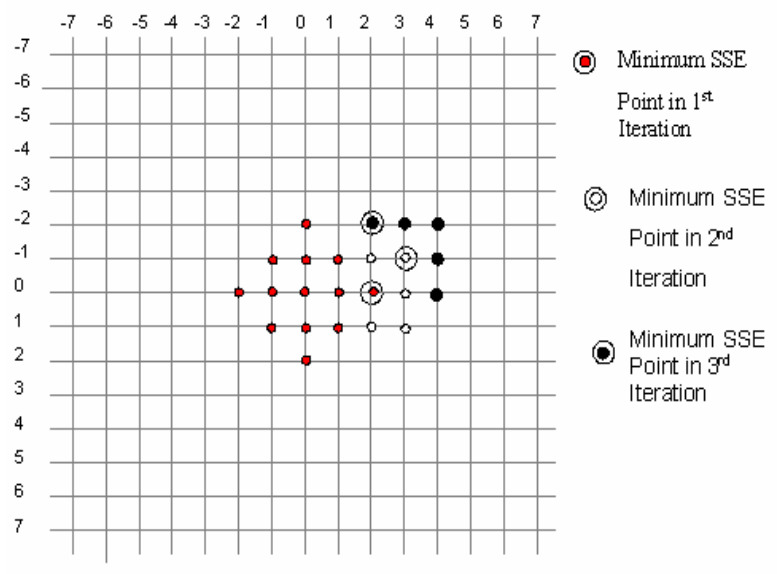

Fig.3. Eight point search pattern

\section{Quality Improvement using Mean Correction:}

In FS method the search is performed by considering all the position inside the reference frame within a search area around the current $\mathrm{MB}$ and testing all of them to find the position that minimizes a suitable cost function. The displacement between the position of the MB in the current frame and the position of the $\mathrm{MB}$, in the reference frame, that minimizes the cost function becomes the motion vector of the
MB. Although FS does find the MVs with minimum SSE but it does not assure minimum residual error, in many cases the minimum SSE as shown in Table VI at location $(1,-4)$ is high, though this is minimum cost function but will lead to degraded PSNR.

The concept of mean correction introduce for the first time in this algorithm will always assure that the residual error is kept at minimum which not only improves PSNR but also helps for early termination of algorithm.

Concept of "mean correction"

Consider the following $8 \times 8$ macroblock.

$$
\begin{aligned}
& 3334333231323334 \\
& 3332333331333233 \\
& 3433323133343333 \\
& 3232343233343334 \\
& 3433323333313332 \\
& 3132333333333334 \\
& 3332333333333434 \\
& 3333333333343434 \\
& \text { (Macroblock A) }
\end{aligned}
$$

\begin{tabular}{|c|}
\hline \\
\hline 3333333333333 \\
\hline 33333333333333 \\
\hline 3333333333333333 \\
\hline 3333333333333333 \\
\hline 3333333333333333 \\
\hline 3333323333323333 \\
\hline $\begin{array}{llllll}33 & 33 & 33 & 33 & 33 & 33\end{array}$ \\
\hline
\end{tabular}

mean of this macroblock is 32.875 which can be rounded off to 33. Now, let us construct an artificial macroblock B with all the values being 33 .

(Macroblock B)

Thus, the SSE is 46. Let us denote this by S1. Now, suppose that our algorithm has found a best match with an $\mathrm{SSE}, \mathrm{S} 2=125$. Clearly, S1 is smaller than S2 and is therefore a better "match". Therefore, instead of sending the motion vector, we can directly send the mean of the current macroblock in such cases. At the receiver end, the macroblock will be reconstructed using this mean value. Therefore this process will assure better PSNR of the reconstructed macroblock using mean value. If $\mathrm{S} 2$ is smaller than $\mathrm{S} 1$, transmit the vector location of S2. It is important to note that the computation of mean itself takes some processing time. Mean computation requires 63 additions, 1 division and 1 rounding off operation. SSE requires 64 subtractions, 64 squaring operations and 63 additions. So a mean computation requires much less computation time than the time required for an SSE computation. Therefore, we have not accounted for it while calculating the search points.

\section{Early Termination of Algorithm:}

To reduce computation in first iteration and iteration to follow without sacrificing the quality of video the algorithm uses technique of early termination using fixed threshold.

In all of the video sequences that we analyzed using the FS, the average PSNR, was in the range of $22 \mathrm{~dB}$ to $45 \mathrm{~dB}$. For early termination of search, selecting PSNR of $22 \mathrm{~dB}$ will degrade the quality and choosing PSNR much above $45 \mathrm{~dB}$ increases the number of search points. Considering this fact, 
we can safely assume that for all practical purposes, a PSNR of 45 can be considered to be a "good PSNR".

$\mathrm{PSNR}=10 * \log (255 / \mathrm{MSE})_{2}$

Therefore, $45=10 * \log (255 / \mathrm{MSE})$

Therefore, $\mathrm{MSE}=2.056$

Therefore, for an $8 \times 8$ macroblock,

$\mathrm{SSE}=64 * 2.056=131$ (approx.)

Thus, we can say that a match is a good match if the SSE is less than or equal to 131 , the threshold SSE. The graph of Fig.4 justifies our selection of fixed threshold $(45 \mathrm{~dB})$ and effect of selecting lower and higher threshold on picture quality and number of search points.

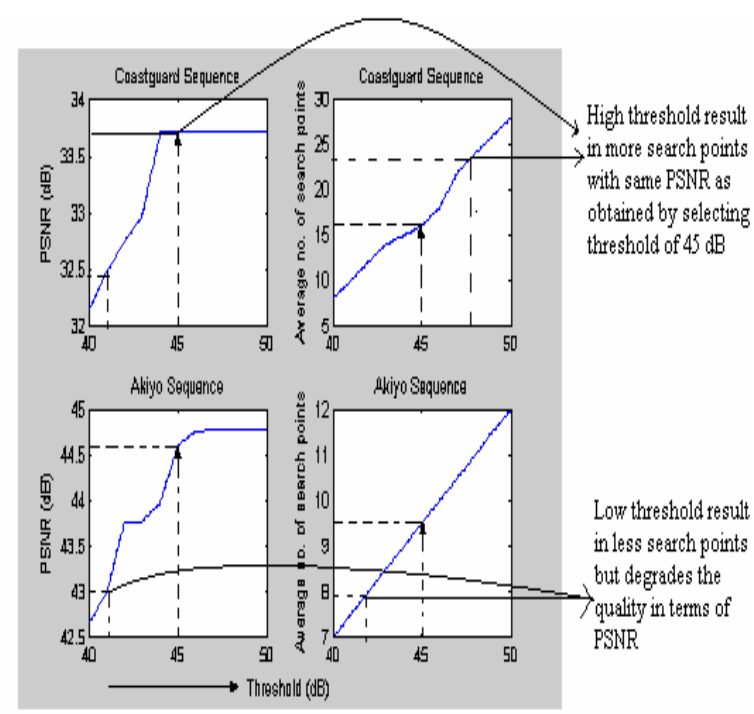

Fig.4 Effect of choice of threshold on picture quality and no. of search points

\section{E. The PBSMC (PBSMCT) Algorithm:}

The flow chart for the aforementioned PBSMC approach is depicted in Fig.5.

1) For the current $\mathrm{MB}$ if $\mathrm{SSE}(\mathrm{S} 1)$ is less than threshold SSE.

a. Consider only the inner 5 points of the central diamond for computation.

b. Obtain the best match $\mathrm{SSE}(\mathrm{S} 2)$ from this five points.

c. If $\mathrm{S} 2<\mathrm{S} 1$, the best match is $\mathrm{S} 2$ transmit vector location of $\mathrm{S} 2$ and go to step 4.

d. If S2 > S1 go tostep3.

2) If SSE (S1) is greater than threshold SSE, consider all 21 search points for the pattern shown in Fig.2. For the first iteration and calculate the SSE at every point proceeding sequentially from the central diamond point to outer points.

Case i) If any of these points has SSE value less than or equal to the threshold SSE transmit vector location of that point and go to step 4 .

Case ii) If match is found in the central diamond region, continue the search by using EPS and find the best match (threshold condition applies) and transmit vector location of that point and go to step 4 .

Case iii) If match is found in the outer 8 points continue the search by using the DS and find the best match (threshold condition applies) and transmit vector location of that point and go to step 4 .

3) Transmit the mean value calculated for the current MB. 4) End.

\section{SIMULATION AND EXPERIMENTAL RESUlT}

The PBSMC algorithm is simulated for both the cases that are with and without threshold using the luminance component of twenty four CIF/QCIF sequence with variable frame numbers

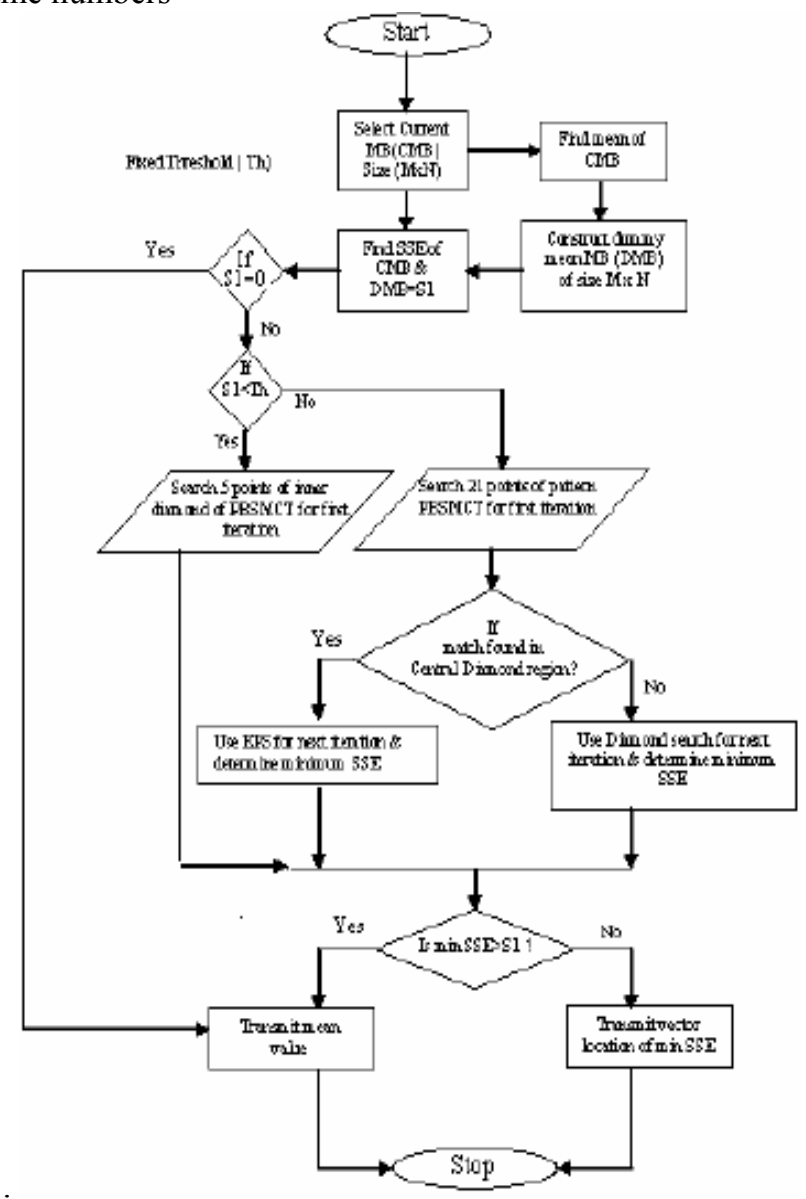

Fig.5. Flowchart of PBSMC (PBSMCT) Algorithm

Out of twenty four sequences, we have listed five video sequences which present different kinds of motion to test the algorithm behavior under different conditions.

1) Miss America: Typical videoconference sequence Fig. 8 with head and shoulder movement with fixed background.

2) Akiyo: Videoconference sequence Fig. 9 with movement restricted to the face area of the speaker and varying background.

3) Foreman: Sequence Fig.10 with large motion in all direction.

4) Garden: Sequence Fig.11 consists mainly of stationary objects, but with a fast camera panning motion.

5) Coastguard: Sequence Fig.12 with large but uniform motion, almost in the horizontal direction.

The average peak signal to noise ratio PSNR of all encoded pictures is used as a measure of objective quality. The sum of square error (SSE) distortion function is used as the block distortion measure (BDM). We compared the PBSMC against four other block based motion estimation 
Methods_-FS, TSS, FSS and DS using the following criteria

1) Failure of sub-optimal algorithm to find true motion vector

TABLE. I. Percentage Motion Vector Probability Distribution Using CIF/QCif SeQuence for Multiple SeQuences

\begin{tabular}{|c|l|l|l|l|l|l|l|l|l|l|l|l|l|l|l|}
\hline $\begin{array}{c}\text { Ver Hor } \\
\text { Ver }\end{array}$ & -7 & -6 & -5 & -4 & -3 & -2 & -1 & 0 & 1 & 2 & 3 & 4 & 5 & 6 & 7 \\
\hline-7 & 0.07 & 0.03 & 0.03 & 0.04 & 0.03 & 0.03 & 0.04 & 0.09 & 0.03 & 0.03 & 0.03 & 0.03 & 0.02 & 0.02 & 0.06 \\
\hline-6 & 0.03 & 0.02 & 0.02 & 0.02 & 0.01 & 0.02 & 0.02 & 0.05 & 0.02 & 0.02 & 0.02 & 0.02 & 0.02 & 0.01 & 0.03 \\
\hline-5 & 0.03 & 0.02 & 0.02 & 0.02 & 0.01 & 0.02 & 0.03 & 0.06 & 0.03 & 0.02 & 0.02 & 0.02 & 0.02 & 0.01 & 0.03 \\
\hline-4 & 0.04 & 0.02 & 0.02 & 0.03 & 0.02 & 0.03 & 0.03 & 0.09 & 0.04 & 0.03 & 0.02 & 0.02 & 0.02 & 0.02 & 0.04 \\
\hline-3 & 0.37 & 0.03 & 0.02 & 0.02 & 0.03 & 0.03 & 0.04 & 0.12 & 0.05 & 0.04 & 0.03 & 0.02 & 0.03 & 0.03 & 0.07 \\
\hline-2 & 2.22 & 0.35 & 0.03 & 0.03 & 0.04 & 0.06 & 0.09 & 0.24 & 0.09 & 0.08 & 0.07 & 0.06 & 0.06 & 0.06 & 0.11 \\
\hline-1 & 0.53 & 1.37 & 0.58 & 0.60 & 0.40 & 0.16 & 0.70 & 2.28 & 0.71 & 0.24 & 0.14 & 0.11 & 0.10 & 0.09 & 0.21 \\
\hline 0 & 0.05 & 1.61 & 2.03 & 2.34 & 1.42 & 1.00 & 5.79 & 54.57 & 3.82 & 1.11 & 0.57 & 0.43 & 0.37 & 0.28 & 0.66 \\
\hline 1 & 0.03 & 0.61 & 0.67 & 0.59 & 0.30 & 0.19 & 0.69 & 2.66 & 0.79 & 0.21 & 0.13 & 0.12 & 0.10 & 0.08 & 0.16 \\
\hline 2 & 0.05 & 0.04 & 0.04 & 0.05 & 0.05 & 0.05 & 0.08 & 0.25 & 0.21 & 0.10 & 0.05 & 0.06 & 0.06 & 0.04 & 0.08 \\
\hline 3 & 0.03 & 0.02 & 0.03 & 0.03 & 0.03 & 0.03 & 0.03 & 0.13 & 0.07 & 0.06 & 0.04 & 0.03 & 0.05 & 0.03 & 0.05 \\
\hline 4 & 0.03 & 0.02 & 0.02 & 0.02 & 0.02 & 0.02 & 0.03 & 0.08 & 0.04 & 0.05 & 0.04 & 0.05 & 0.05 & 0.05 & 0.06 \\
\hline 5 & 0.03 & 0.02 & 0.02 & 0.02 & 0.02 & 0.02 & 0.02 & 0.06 & 0.03 & 0.05 & 0.04 & 0.02 & 0.04 & 0.06 & 0.07 \\
\hline 6 & 0.03 & 0.02 & 0.02 & 0.02 & 0.02 & 0.02 & 0.02 & 0.06 & 0.02 & 0.02 & 0.02 & 0.01 & 0.02 & 0.03 & 0.04 \\
\hline 7 & 0.05 & 0.03 & 0.03 & 0.03 & 0.03 & 0.02 & 0.02 & 0.08 & 0.03 & 0.02 & 0.02 & 0.02 & 0.02 & 0.02 & 0.06 \\
\hline
\end{tabular}

TABle. II Percentage Of Times Three-Step Search Have Failed To Find Best Match That Full Search Has Found At Particular Point WITHIN 15 X 15 SEARCH WINDOW FOR 75 FRAMES OF AKIYO SEQUENCE.

\begin{tabular}{|c|c|c|c|c|c|c|c|c|c|c|c|c|c|c|c|}
\hline & -7 & -6 & -5 & -4 & -3 & -2 & -1 & 0 & 1 & 2 & 3 & 4 & 5 & 6 & 7 \\
\hline-7 & 70.2 & 74.72 & 80.91 & 44.73 & 74.63 & 72.66 & 76.94 & 56.13 & 68.62 & 72.8 & 70.62 & 69.77 & 74.1 & 74.12 & 73.96 \\
\hline-6 & 81.94 & 65.27 & 75.2 & 53.06 & 71.19 & 56.02 & 80.48 & 45.7 & 70.45 & 58.33 & 78.64 & 62.36 & 75 & 65.18 & 74.94 \\
\hline-5 & 74.7 & 77.37 & 48.96 & 44.41 & 59.17 & 64.06 & 71.49 & 32.45 & 60.84 & 69.04 & 63.19 & 48.49 & 59.63 & 67.09 & 72.13 \\
\hline-4 & 76.91 & 59.85 & 66.58 & $\mathbf{0}$ & 54.4 & 53.9 & 62.34 & $\mathbf{0}$ & 56.59 & 51.45 & 57.7 & $\mathbf{0}$ & 56.68 & 57.87 & 76.2 \\
\hline-3 & 69.49 & 69.04 & 67.52 & 56.8 & 36.65 & 58.43 & 65.11 & 24.5 & 54.72 & 53.05 & 56.37 & 52.76 & 57.91 & 65.78 & 68.84 \\
\hline-2 & 77.07 & 58.7 & 69.9 & 46.58 & 60.4 & 13.92 & 67.01 & 16.75 & 54.35 & 24.41 & 55.66 & 54.45 & 59.17 & 50.39 & 69.39 \\
\hline-1 & 73.84 & 59.82 & 34.14 & 22.95 & 38.29 & 69.19 & 19.72 & 11.74 & 20.43 & 54.98 & 50.27 & 51.35 & 53.83 & 66.38 & 76.71 \\
\hline 0 & 60.25 & 53.44 & 15.29 & $\mathbf{0}$ & 16.12 & 14.64 & 8.72 & $\mathbf{0}$ & 11.14 & 21.3 & 23.74 & $\mathbf{0}$ & 23.23 & 37.82 & 46.55 \\
\hline 1 & 76.54 & 67.85 & 45.31 & 22.85 & 35.05 & 61.5 & 17.56 & 12.95 & 22.85 & 67.57 & 65.14 & 60.22 & 59.14 & 70.18 & 79.36 \\
\hline 2 & 78.83 & 66.33 & 75.61 & 56.18 & 67.25 & 17.01 & 58.4 & 18.44 & 46.19 & 22.29 & 56.5 & 51.1 & 56.71 & 56.11 & 70.17 \\
\hline 3 & 74.15 & 76.1 & 65.33 & 50.85 & 56.8 & 53.52 & 57.56 & 24.78 & 47.87 & 47.54 & 37.5 & 48.18 & 40.79 & 54.3 & 65.09 \\
\hline 4 & 77.15 & 65.58 & 61.95 & $\mathbf{0}$ & 60.96 & 51.88 & 56.42 & $\mathbf{0}$ & 57.21 & 47.89 & 42.01 & $\mathbf{0}$ & 29.37 & 41.74 & 65.26 \\
\hline 5 & 75.83 & 74.63 & 62.8 & 44.65 & 61.09 & 67.12 & 64.2 & 28.84 & 65.5 & 54.98 & 42.55 & 49.58 & 29.41 & 55.58 & 61.69 \\
\hline 6 & 80.4 & 68.38 & 80.78 & 57.78 & 78.06 & 65.65 & 78.73 & 51.73 & 78.7 & 52.96 & 63.94 & 56.64 & 67.17 & 46.5 & 68.17 \\
\hline 7 & 73.76 & 75.32 & 88.25 & 60.78 & 79.75 & 70.66 & 75 & 55.73 & 78.19 & 73.96 & 79.81 & 68.03 & 75.18 & 78.33 & 65.23 \\
\hline
\end{tabular}

TABle. III Percentage Of Times Four-Step Search Have Failed To Find Best Match That Full Search Has Found At Particular Point WITHIN 15 X 15 SEARCH WINDOW FOR 75 FRAMES OF AKIYO SEQUENCE

\begin{tabular}{|c|c|c|c|c|c|c|c|c|c|c|c|c|c|c|c|}
\hline & -7 & -6 & -5 & -4 & -3 & -2 & -1 & 0 & 1 & 2 & 3 & 4 & 5 & 6 & 7 \\
\hline-7 & 84.35 & 86.91 & 84.69 & 86.01 & 81.59 & 76.35 & 78.25 & 65.87 & 74.5 & 76.13 & 80.57 & 83.63 & 86.41 & 87.33 & 85.67 \\
\hline-6 & 90.74 & 83.68 & 96.4 & 80.82 & 95.34 & 71.78 & 95.72 & 64.9 & 97.1 & 72.84 & 96.59 & 83.65 & 97.18 & 89.73 & 87.34 \\
\hline-5 & 86.02 & 98.17 & 85.67 & 88.18 & 83.49 & 79.69 & 88.26 & 76.92 & 85.62 & 83.29 & 87.83 & 89.63 & 91.48 & 97.44 & 84.07 \\
\hline-4 & 85.45 & 82.2 & 92.09 & 53.5 & 77.36 & 54.16 & 78.34 & 37.44 & 69.43 & 48.94 & 77.02 & 61.48 & 89.32 & 79.53 & 87.54 \\
\hline-3 & 78.84 & 94.31 & 85.19 & 75.2 & 39.96 & 51.61 & 59.21 & 25.77 & 53.41 & 40.68 & 57.88 & 75.59 & 82.24 & 97.34 & 77.6 \\
\hline-2 & 77.96 & 67.63 & 86.87 & 46.8 & 55.78 & 0 & 60.6 & $\mathbf{0}$ & 42.24 & $\mathbf{0}$ & 39.42 & 36.97 & 78.63 & 61.79 & 76.07 \\
\hline-1 & 78.52 & 93.32 & 75.59 & 52.97 & 42.25 & 63.03 & 14.6 & 9.05 & 12.88 & 36.63 & 43.92 & 65.32 & 82.16 & 96.75 & 81.44 \\
\hline 0 & 66.43 & 64.83 & 67.13 & 38.49 & 19.01 & 0 & 6.51 & 0 & 8.01 & 0 & 24.87 & 35.69 & 67.85 & 48.98 & 52.72 \\
\hline 1 & 82.41 & 96.61 & 82.08 & 61.3 & 44.13 & 52.09 & 13.7 & 11.21 & 17.46 & 56.31 & 60.72 & 71.06 & 82.99 & 96.39 & 80.32 \\
\hline 2 & 84.19 & 77.72 & 87.95 & 59.24 & 63.22 & 0 & 45.05 & 0 & 30.9 & 0 & 46.13 & 27.48 & 73.5 & 50.57 & 58.21 \\
\hline 3 & 82.64 & 94.78 & 87.19 & 74.94 & 65.31 & 49.47 & 55.04 & 24.68 & 38.86 & 32.57 & 41.83 & 65.64 & 73.55 & 95.43 & 75.26 \\
\hline 4 & 87.33 & 89.02 & 94.39 & 64.86 & 79.41 & 53.62 & 72.04 & 36.29 & 74.88 & 35.36 & 65.28 & 45.95 & 79.85 & 62.67 & 77.07 \\
\hline 5 & 88.03 & 95.22 & 91.67 & 88.68 & 89.08 & 84.75 & 86.11 & 73.68 & 84.99 & 69.05 & 60.47 & 82.82 & 76.83 & 95.69 & 72.5 \\
\hline 6 & 90.45 & 89.34 & 98.7 & 83.23 & 98.39 & 77.1 & 96.64 & 67.89 & 93.77 & 64.51 & 97.4 & 82.74 & 94.34 & 70.56 & 81.98 \\
\hline 7 & 86.94 & 87.98 & 93.61 & 87.17 & 85.93 & 80.84 & 80.1 & 65.6 & 82.63 & 78.39 & 85.9 & 84.01 & 84.67 & 89.17 & 79.98 \\
\hline
\end{tabular}

TABLE. IV Percentage Of Times Diamond Search Have Failed To Find Best Match That Full Search Has Found At Particular Point Within 15 X 15 SEARCH Window For 75 Frames OF AKIYO SEQUENCE. 


\begin{tabular}{|c|c|c|c|c|c|c|c|c|c|c|c|c|c|c|c|}
\hline & -7 & -6 & -5 & -4 & -3 & -2 & -1 & 0 & 1 & 2 & 3 & 4 & 5 & 6 & 7 \\
\hline-7 & 81.91 & 81.72 & 80.53 & 74.09 & 77.36 & 77.09 & 76.44 & 66.96 & 73.21 & 78.86 & 81.52 & 83.38 & 84.87 & 87.06 & 80.2 \\
\hline-6 & 89.16 & 86.19 & 84.8 & 81.22 & 80.93 & 82.57 & 83.96 & 68.2 & 81.27 & 77.47 & 86.69 & 87.83 & 91.94 & 90.63 & 85.86 \\
\hline-5 & 86.27 & 91.13 & 77.91 & 80.83 & 75.69 & 74.06 & 76.31 & 64.5 & 69.47 & 75.89 & 83.48 & 82.94 & 87.41 & 88.89 & 84.54 \\
\hline-4 & 81.76 & 84.47 & 87.24 & 65.42 & 78.93 & 66.5 & 65.16 & 43.35 & 60.81 & 63.39 & 75.98 & 75.68 & 82.79 & 87.4 & 84.19 \\
\hline-3 & 76.61 & 81.85 & 76.64 & 77.33 & 47.88 & 62.05 & 43.96 & 26.1 & 41.86 & 50.96 & 59.83 & 79.53 & 79.08 & 79.28 & 79.36 \\
\hline-2 & 76.04 & 73.67 & 69.9 & 56.95 & 58.82 & 24.27 & 38.31 & $\mathbf{0}$ & 23.56 & 20.83 & 44.86 & 50.74 & 63.21 & 66.6 & 77.35 \\
\hline-1 & 75.26 & 69.81 & 61.56 & 51.43 & 32.88 & 36.53 & $\mathbf{0}$ & 7.14 & $\mathbf{0}$ & 23.12 & 32.02 & 53.58 & 58.27 & 65.37 & 65.11 \\
\hline 0 & 69.31 & 67.57 & 53.83 & 42.42 & 18.11 & $\mathbf{0}$ & 4.17 & $\mathbf{0}$ & 5.79 & $\mathbf{0}$ & 24.44 & 38.94 & 46.05 & 51.59 & 54.67 \\
\hline 1 & 70.53 & 70.19 & 62.81 & 50.16 & 35.41 & 35.66 & $\mathbf{0}$ & 6.7 & $\mathbf{0}$ & 26.37 & 34.23 & 49.81 & 54.52 & 64.95 & 64.82 \\
\hline 2 & 83.71 & 82.75 & 80.92 & 66.75 & 64.36 & 33.41 & 29.75 & $\mathbf{0}$ & 16.6 & 14.8 & 48.87 & 37.2 & 53.76 & 58.24 & 61.92 \\
\hline 3 & 82.08 & 90.93 & 83.17 & 77.13 & 64.91 & 59.91 & 43.02 & 24.17 & 25.43 & 40.73 & 45.67 & 63.15 & 57.6 & 72.03 & 72.25 \\
\hline 4 & 87.1 & 91.69 & 88.29 & 80.83 & 77.54 & 64.35 & 65.74 & 41.93 & 56.89 & 44.46 & 62.5 & 54.05 & 65.78 & 63.02 & 72.15 \\
\hline 5 & 86.03 & 90.81 & 87.2 & 87.42 & 84.64 & 76.95 & 70.99 & 56 & 72.71 & 51.46 & 54.1 & 72.39 & 63.46 & 72.15 & 70.04 \\
\hline 6 & 88.19 & 89.34 & 91.21 & 88.02 & 83.23 & 85.11 & 82.09 & 70.9 & 81.56 & 71.55 & 78.44 & 86.28 & 85.28 & 71.73 & 77.18 \\
\hline 7 & 80.59 & 87.34 & 89.9 & 85.69 & 76.54 & 79.64 & 77.81 & 65.91 & 79.73 & 79.43 & 85.9 & 82.53 & 85.4 & 88.33 & 79.35 \\
\hline
\end{tabular}

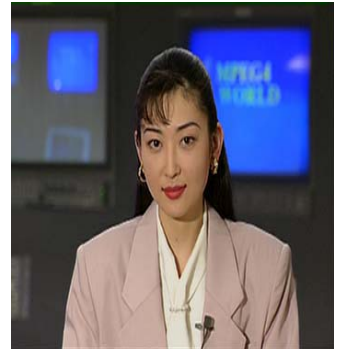

Fig. 6 Miss America Sequence

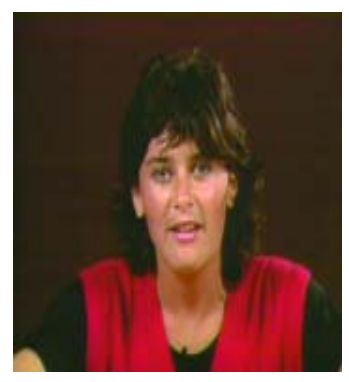

Fig.7 Akiyo sequence

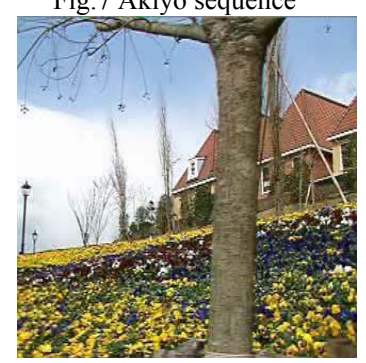

Fig.8 Foreman sequence

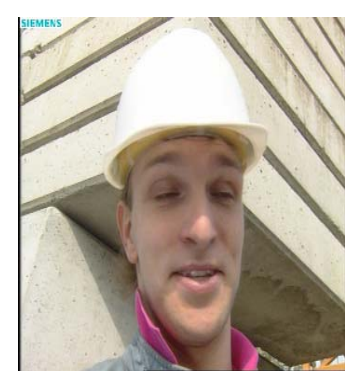

Fig. 9Garden sequence

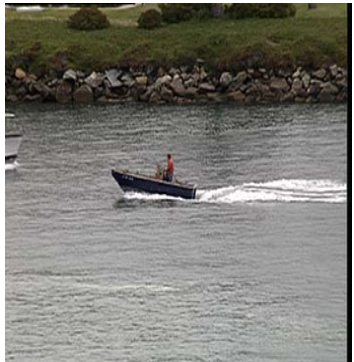

Fig.10 Coastguard sequence

We know that TSS, FSS, and DS algorithm have inherent problem in their search pattern due to which these algorithms have poor PSNR as compared to FS. In order to analyze the shortcomings in the search pattern of these algorithms we have considered three cases i) MV located in central diamond region. ii) MV located in proximity of central diamond region. iii) MV located at the edge of search window. The carphone sequence is considered for the first case, the SSE values of which are derived using FS for frame 45 for MB at $(15,1)$ and are depicted in Table V. As FS method computes SSE at all points in search window of $15 \times 15$, it is very easy to see that minimum SSE is at location $(-1,0)$, but TSS is not able to find the minimum value instead gets trapped in to local minima at location $(4,4)$ and finds minimum SSE point at $(5,5)$, which result in large residual error and poor PSNR, FSS similar to TSS and DS similar to TSS gets trapped in to local minima at $(2,2)$ and $(1,1)$ and finds minimum SSE point at locations $(5,5),(0,1)$ respectively resulting in poor PSNR. In other words due to its static search pattern TSS, FSS and DS fails to locate the minimum SSE point located at $(-1,0)$ in central diamond region, but the above limitation of getting trapped in local minima is overcome by PBSMC algorithm which finds the minimum SSE point at location $(-1,0)$ in very first iteration.

The search patterns of TSS, FSS, DS and PBSMC to locate minimum SSE point for case(i) is as tabulated in Table VI. The first iteration for TSS and FSS as shown in first column is comparison, in terms of SSE at nine different locations with step size of \pm 4 pixels and \pm 2 pixel respectively from the center point. The minimum cost function (SSE) out of this nine point becomes center point for the next iteration, in this case locations $(4,4),(2,2)$ are minimum SSE points for TSS and FSS in first iteration. The process to find minimum SSE 
will continues till the step size is reduced to \pm 1 pixel. The search sequence in case of DS is also nine different points forming diamond shape with search location as shown in first column of Table VI, the further steps of DS algorithm to locate minimum SSE points are almost similar to FSS. As can be seen TSS and DS converges to minimum SSE value in third iteration with 25 maximum search points and FSS converges to minimum SSE value in fourth iteration with maximum of 33 search points, the important point to note here is that these three algorithms uses considerable search points, still are unable to find true MV located at location $(-1,0)$. Our algorithm PBSMC due to its adaptive search pattern takes only few points to track true motion vector that FS has searched.

As we have seen the limitation of case(i) now let us analyze case(ii), the sequence under consideration is stefan, which is large motion sequence involving camera panning and zooming movements, the $\mathrm{MB}$ for comparison of minimum cost function is located at $(29,8)$ for frame number 45. SSE value for all the points within search window of $15 \times 15$ using full search method is computed and is as shown in Table VII, the minimum SSE is found at location $(1,-4)$ which is between central point $(0,0)$ and edge of the search window. Here FSS and DS gets trapped at $(0,-2)$ and finds minimum SSE point of value at location $(0,-3)$, whereas TSS miserably gets misguided and travels in opposite direction to locate minimum SSE point at location $(-7,3)$ which result in poor PSNR, but PBSMC algorithm in fourth iteration finds minimum SSE point at same point which FS has found.

As mentioned earlier that the sub optimal algorithms are not efficient to find true $\mathrm{MV}$, in other words if TSS can track large motion it is unable to track low motion whereas FSS and DS can track low motion but are unable to track large motion although DS performs better than FSS in the vicinity of central point but fails to track MV located at edge of the window. The following example which considers third case with frame number 128 of bus sequence, the MB placed at $(9,21)$ has minimum SSE at $(5,-5)$ which is found by using FS method. As shown in Table VIII, the FSS and DS totally gets misguided and are unable to track true motion vector which is located at $(5,-5)$ instead FSS finds minimum SSE value at location $(-2,7)$ and DS finds minimum SSE value at location $(-2,6)$ both of which are in opposite direction as compared to minimum SSE vector location $(5,-5)$, TSS comes closer $(1,-5)$ to vector location that FS has found $(5,-5)$ but still is unable to detect the true motion vector. It is important to note that our algorithm tracks the true motion vector in fourth iteration due to search points located in outer diamond region which justify our selection of additional search points in outer diamond.

The Search sequence of TSS, FSS, DS and PBSMC in Table VIII is tabulated in Table IX, along with their minimum SSE point for third cases that is minimum SSE point located at the edge of search window. As can be seen from Table IX that TSS converges to minimum SSE value in third iteration with 25 maximum search points at location $(5,5)$ and FSS and DS converges to minimum SSE value in fourth iteration with maximum of 33 search points. Again these three algorithms fail to find true MV located at location $(-1,0)$. Our algorithm PBSMC due to its intelligent search pattern takes only few points to track true motion vector that FS has searched. It can be concluded from above observation that TSS algorithm is unable to detect motion vector in proximity of central diamond point and FSS and DS are unable to detect motion vector located at the edge of search window but with PBSMC we are able to track almost all MVs that is MVs located in the central diamond region, in proximity of central diamond point or at the edge of the search window.

Tables X and X1 summarizes the PSNR value and average number of search points for algorithms like FS, probability based search with mean correction (PBSMC), probability based search with mean correction and threshold (PBSMCT), DS, FSS, and TSS. Simulation result shows considerable improvement in PSNR of sequences like suzie, carphone, mother and daughter, silent, tempete and waterfall.

TABLE. V FAILURE Of TSS, FSS, AND DS SEARCh PATTERns To LOCATE Minimum SSE POINT In CENTRAL DiAMOND REGION FOR CARPHONE SEQUENCE, Frame 4, ROW-15, COL-11

\begin{tabular}{|c|c|c|c|c|c|c|c|c|c|c|c|c|c|c|c|}
\hline & -7 & -6 & -5 & -4 & -3 & -2 & -1 & 0 & 1 & 2 & 3 & 4 & 5 & 6 & 7 \\
\hline-7 & 4255 & 919 & 4645 & 6697 & 7588 & 7920 & 13555 & 31224 & 59399 & 93597 & 133642 & 178359 & 228128 & 280061 & 330412 \\
\hline-6 & 4455 & 4406 & 3409 & 4757 & 5692 & 6401 & 11593 & 28582 & 57652 & 98205 & 147048 & 202957 & 265198 & 328758 & 389977 \\
\hline-5 & 3944 & 4351 & 4871 & 4934 & 5082 & 6030 & 12265 & 28798 & 57327 & 99158 & 153395 & 218320 & 289798 & 363243 & 433032 \\
\hline-4 & 4072 & 3763 & 3896 & 4300 & 4429 & 4216 & 10609 & 26711 & 53245 & 93849 & 148910 & 217923 & 295717 & 381436 & 463323 \\
\hline-3 & 4334 & 3804 & 3627 & 3521 & 3432 & 723 & 2665 & 17550 & 45068 & 85135 & 139056 & 209140 & 287847 & 377169 & 465302 \\
\hline-2 & 4569 & 4169 & 3828 & 3473 & 3656 & 3162 & 201 & 5014 & 23701 & 55345 & 99324 & 159628 & 230121 & 313606 & 405815 \\
\hline-1 & 5013 & 4442 & 4074 & 3724 & 3408 & 3357 & 2542 & 99 & 7188 & 29486 & 64411 & 114008 & 175004 & 249796 & 337246 \\
\hline 0 & 5413 & 4625 & 4438 & 3975 & 3364 & 3065 & 3479 & 2204 & 282 & 10191 & 35561 & 74917 & 125502 & 189687 & 268627 \\
\hline 1 & 5293 & 4774 & 4640 & 4347 & 3670 & 3376 & 3220 & 3390 & 1472 & 967 & 13388 & 41042 & 79795 & 131766 & 197125 \\
\hline 2 & 5384 & 4840 & 4639 & 4421 & 3815 & 3471 & 3271 & 3081 & 3107 & 1052 & 2014 & 16094 & 42625 & 82669 & 136297 \\
\hline 3 & 5406 & 5061 & 4889 & 4289 & 3893 & 3719 & 3345 & 3249 & 3122 & 3163 & 826 & 3013 & 16754 & 44445 & 86988 \\
\hline 4 & 5296 & 5134 & 4932 & 4507 & 4102 & 3821 & 3705 & 3404 & 3367 & 3374 & 3241 & 593 & 3843 & 18914 & 49413 \\
\hline 5 & 5274 & 4890 & 4690 & 4474 & 4175 & 3741 & 3538 & 3503 & 3469 & 3432 & 3528 & 2709 & 541 & 5388 & 24042 \\
\hline 6 & 5103 & 4784 & 4507 & 4317 & 4169 & 3781 & 3659 & 3723 & 3739 & 3857 & 3675 & 3521 & 2468 & 569 & 9083 \\
\hline 7 & 5045 & 4859 & 4539 & 4517 & 4275 & 3841 & 3933 & 3827 & 4053 & 3892 & 3908 & 3725 & 3626 & 1788 & 1534 \\
\hline
\end{tabular}


TABLE. VI SEARCh ITERATION AND Minimum SSE VALUE FOR TSS, FSS, DS AND PBMCT ALGORITHM FOR CARPhONE SEQUENCE, FRAME-4 Row-15, COL-11

\begin{tabular}{|c|c|c|c|c|c|}
\hline Sr.no & Algorithm & \multicolumn{4}{|c|}{ Search points in different iterations with minimum SSE value } \\
\hline & & $1^{\text {st }}$ iteration & $2^{\text {nd }}$ iteration & $3^{\text {rd }}$ iteration & $4^{\text {th }}$ iteration \\
\hline 1 & TSS & $\begin{array}{l}(0,0)(-4,0)(-4,-4) \\
(-4,4)(0,-4)(4,-4) \\
(4,0)(4,4)(0,4) \\
{[\text { SSE-593 }(4,4)]}\end{array}$ & $\begin{array}{l}(2,2)(2,4)(2,6) \\
(4,2)(4,0)(4,6) \\
(6,2)(6,4)(6,6) \\
{[\text { SSE-569 }(6,6)]}\end{array}$ & $\begin{array}{l}(5,5)(5,6)(5,7) \\
(6,5)(6,6)(6,7) \\
(7,5)(7,6)(7,7) \\
{[S S E-} \\
541(5,5)]\end{array}$ & \\
\hline 2 & FSS & $\begin{array}{l}(0,0)(-2,0)(-2,-2) \\
(-2,2)(0,-2)(0,2) \\
(2,-2)(2,0)(2,2) \\
{[\text { SSE-1052 }(2,2)]}\end{array}$ & $\begin{array}{l}(0,0)(0,2)(0,4) \\
(2,0)(2,2)(2,4) \\
(4,0)(4,2)(4,4) \\
{[\text { SSE-593(4,4)] }}\end{array}$ & $\begin{array}{l}(2,2)(2,4)(2,6) \\
(4,2)(4,4)(4,6) \\
(6,2)(6,4)(6,6) \\
{[\text { SSE- }} \\
569(6,6)]\end{array}$ & $\begin{array}{l}(5,5)(5,6)(5,7) \\
(6,5)(6,6)(6,7) \\
(7,5)(7,6)(7,7) \\
{[\text { SSE- }} \\
541(5,5)]\end{array}$ \\
\hline 3 & DS & $\begin{array}{l}(0,0)(-2,0)(-1,-1) \\
(-1,1)(0,-2)(0,2) \\
(1,-1)(1,1)(2,0) \\
{[\text { SSE-1472(1,1)] (Local minima) }}\end{array}$ & $\begin{array}{l}(-1,1)(0,-1)(0,1) \\
(1,-1)(1,1)(1,3) \\
(2,0)(2,2)(3,1) \\
{[\text { SSE-1472(1,1)] }}\end{array}$ & $\begin{array}{l}(0,0)(0,1)(0,2) \\
(1,0)(1,1)(1,2) \\
(2,0)(2,1)(2,2) \\
{[\text { SSE- }} \\
282(0,1)] \\
\end{array}$ & \\
\hline 4 & $\begin{array}{l}\text { PBSMC } \\
\text { (PBSMCT) }\end{array}$ & $\begin{array}{l}(0,0)(0,-1)(0,-2)(0,1) \\
(0,2)(-1,0)(-1,-1)(-1,1) \\
(-2,0)(1,0)(1,-1)(1,1) \\
(2,0)(-6,0)(6,0)(-3,-3) \\
(-3,3)(3,-3)(3,3)(0,-6) \\
(0,6)[\text { SSE- } 99(-1,0)]\end{array}$ & \multicolumn{3}{|c|}{$\begin{array}{l}\text { IF S1=0 search iteration are not needed. } \\
\text { IF S1< TH search inner } 5 \text { points }(0,0)(0,-1)(0,1) \\
(-1,0)(1,0) \text {. } \\
\text { IF S1> TH search all } 21 \text { points }\end{array}$} \\
\hline
\end{tabular}

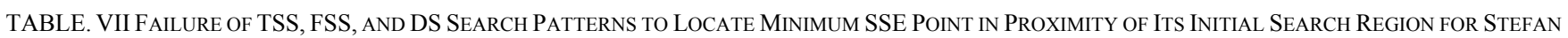
SEQUENCE, FRAME 45, ROW-28, COL-8

\begin{tabular}{|c|c|c|c|c|c|c|c|c|c|c|c|c|c|c|c|}
\hline & -7 & -6 & -5 & -4 & -3 & -2 & -1 & 0 & 1 & 2 & 3 & 4 & 5 & 6 & 7 \\
\hline-7 & 25583 & 25879 & 25392 & 24973 & 25024 & 24892 & 26150 & 28272 & 26771 & 19490 & 12575 & 13215 & 23481 & 32110 & 36829 \\
\hline-6 & 25764 & 25914 & 25668 & 25910 & 25789 & 26153 & 26559 & 23637 & 17593 & 13637 & 19181 & 29162 & 32172 & 30642 & 42376 \\
\hline-5 & 25050 & 25257 & 25204 & 25454 & 25536 & 25892 & 22824 & 15836 & 13215 & 22366 & 38628 & 46853 & 41298 & 35311 & 46953 \\
\hline-4 & 24395 & 24420 & 24088 & 23533 & 24121 & 24906 & 22769 & 19798 & 20584 & 28877 & 39174 & 45274 & 50464 & 54866 & 58714 \\
\hline-3 & 24698 & 24515 & 23914 & 22966 & 24309 & 26658 & 26275 & 25077 & 24603 & 26758 & 35369 & 48064 & 63793 & 77234 & 83100 \\
\hline-2 & 24533 & 24311 & 25613 & 28335 & 29221 & 27133 & 27687 & 34756 & 38349 & 38038 & 43674 & 53929 & 65304 & 80819 & 100205 \\
\hline-1 & 23986 & 25304 & 29864 & 31416 & 23756 & 14702 & 13138 & 30895 & 53746 & 68263 & 73814 & 75614 & 78370 & 93906 & 120299 \\
\hline 0 & 25209 & 27866 & 28641 & 20018 & 11239 & 13044 & 19071 & 32219 & 56520 & 87845 & 108711 & 117530 & 120182 & 132104 & 144522 \\
\hline 1 & 26796 & 25401 & 17377 & 10479 & 20078 & 44048 & 65925 & 83090 & 97309 & 117584 & 136440 & 152192 & 167763 & 176026 & 167742 \\
\hline 2 & 24942 & 20022 & 15723 & 24046 & 50501 & 83356 & 112218 & 140275 & 154293 & 160607 & 160489 & 162161 & 169776 & 175800 & 165370 \\
\hline 3 & 22349 & 19854 & 28226 & 46624 & 69385 & 93127 & 118947 & 152717 & 177897 & 189814 & 180117 & 163170 & 153913 & 156655 & 151166 \\
\hline 4 & 24001 & 26914 & 42598 & 55870 & 64955 & 80609 & 103047 & 134671 & 164004 & 182292 & 175382 & 162913 & 161541 & 164673 & 154691 \\
\hline 5 & 31539 & 35627 & 48284 & 59347 & 72397 & 92895 & 113031 & 133676 & 153708 & 168502 & 162936 & 158592 & 165170 & 165585 & 149119 \\
\hline 6 & 36276 & 36785 & 44159 & 64326 & 95361 & 125007 & 147484 & 167556 & 183691 & 192413 & 183517 & 173912 & 169304 & 158416 & 135781 \\
\hline 7 & 31911 & 26693 & 31490 & 53072 & 83013 & 109511 & 134787 & 157981 & 172922 & 179097 & 168068 & 150823 & 137565 & 124837 & 103309 \\
\hline
\end{tabular}

TABLE. ViII Failure of TSS, FSS, and DS Search Patterns to Locate Minimum SSE Point Closer to Edges of Search Window for Bus SEQUENCE, FRAME 128, COL-9, COL-21.

\begin{tabular}{|c|c|c|c|c|c|c|c|c|c|c|c|c|c|c|c|}
\hline & -7 & -6 & -5 & -4 & -3 & -2 & -1 & 0 & 1 & 2 & 3 & 4 & 5 & 6 & 7 \\
\hline-7 & 105324 & 57114 & 7014 & 15421 & 98009 & 192661 & 226532 & 223813 & 226121 & 199138 & 143396 & 106323 & 99960 & 94692 & 91818 \\
\hline-6 & 106727 & 58023 & 7174 & 14953 & 97239 & 193232 & 228183 & 224768 & 225351 & 197830 & 142790 & 106299 & 100286 & 96003 & 94398 \\
\hline-5 & 107535 & 58227 & 7012 & 14769 & 96541 & 193815 & 230307 & 226461 & 225349 & 196707 & 141783 & 104495 & 97761 & 94587 & 94069 \\
\hline-4 & 107519 & 57532 & 6566 & 15003 & 97226 & 194977 & 231202 & 226140 & 224418 & 195835 & 140566 & 102195 & 95317 & 93394 & 93127 \\
\hline-3 & 107392 & 57283 & 6613 & 15185 & 97789 & 195684 & 231135 & 225271 & 223306 & 195221 & 139800 & 100826 & 93586 & 91651 & 91202 \\
\hline-2 & 107986 & 57379 & 6677 & 15246 & 96866 & 194496 & 231635 & 226881 & 223545 & 194131 & 139238 & 100793 & 93406 & 91208 & 89338 \\
\hline-1 & 108451 & 56879 & 6260 & 15117 & 95802 & 193229 & 232307 & 228483 & 223255 & 192142 & 138764 & 102964 & 97676 & 95620 & 90581 \\
\hline 0 & 107314 & 55970 & 5933 & 14892 & 95136 & 193387 & 233506 & 229977 & 223601 & 192053 & 139979 & 106219 & 102027 & 98480 & 90686 \\
\hline 1 & 106250 & 55421 & 5890 & 15253 & 95518 & 194126 & 235397 & 232503 & 225740 & 193928 & 141724 & 107437 & 100753 & 95039 & 88083 \\
\hline 2 & 106018 & 55100 & 5744 & 16149 & 96380 & 194721 & 236503 & 233640 & 226926 & 194494 & 141433 & 106235 & 98273 & 91945 & 86182 \\
\hline 3 & 105209 & 54662 & 5702 & 16775 & 96955 & 195581 & 237306 & 233890 & 226986 & 193798 & 141265 & 107479 & 101030 & 94736 & 88710 \\
\hline 4 & 103441 & 53621 & 5412 & 16926 & 95846 & 193868 & 236827 & 234817 & 227605 & 193226 & 142378 & 110615 & 104081 & 96607 & 90747 \\
\hline 5 & 101952 & 52471 & 5271 & 17460 & 94300 & 190990 & 236306 & 236943 & 230375 & 195032 & 144870 & 112828 & 105316 & 97381 & 91727 \\
\hline 6 & 99315 & 50974 & 5598 & 18668 & 93886 & 189370 & 235775 & 237693 & 232154 & 197029 & 147101 & 113383 & 104498 & 96748 & 92879 \\
\hline 7 & 92881 & 48162 & 7137 & 22128 & 94389 & 184221 & 227503 & 229034 & 223906 & 190882 & 146692 & 115247 & 104428 & 96422 & 94897 \\
\hline
\end{tabular}

The PSNR value of this sequences are even better than full search with less number of search points, this is because of mean correction technique used to improve the quality of the picture. Other sequences like akiyo, coastguard, container, etc PSNR is comparable to FS but far better than TSS, FSS, and DS. Algorithm like PBSMC uses almost same number of search points that TSS uses but PSNR values are better than TSS, FSS and DS algorithm. 
TABLE. IX SEARCh ITERATION AND MINIMUM SSE VALUE FOR TSS, FSS, DS AND PBSMCT ALGORITHM FOR BUS SEQUENCE, FRAME-128 Row-9, COL-21

\begin{tabular}{|c|c|c|c|c|c|}
\hline \multirow{2}{*}{$\begin{array}{l}\text { Sr.n } \\
0\end{array}$} & \multirow[t]{2}{*}{ Algorithm } & \multicolumn{4}{|c|}{ Search points in different iterations with minimum SSE value } \\
\hline & & $1^{\text {st }}$ iteration & $2^{\text {nd }}$ iteration & $3^{\text {ra }}$ iteration & $4^{\text {th }}$ iteration \\
\hline 1 & TSS & $\begin{array}{l}(0,0)(-4,0)(-4,-4) \\
(-4,4)(0,-4)(4,-4) \\
(4,0)(4,4)(0,4) \\
{[\text { SSE-14892 }(0,-4)]}\end{array}$ & $\begin{array}{l}(-2,-6)(-2,-4)(-2,-2) \\
(0,-6)(0,-4)(0,-2) \\
(2,-6)(2,-4)(2,-2) \\
{[S S E-14892(0,-4)]}\end{array}$ & $\begin{array}{l}(-1,-5)(-1,-4)(-1,-3) \\
(0,-5)(0,-4)(0,-3) \\
(1,-5)(1,-4)(1,-3) \\
{[\text { SSE-5890(1,-5)] }}\end{array}$ & \\
\hline 2 & FSS & $\begin{array}{l}(-2,-2)(-2,0)(-2,0) \\
(0,-2)(0,0)(0,2) \\
(2,-2)(2,0)(2,2) \\
{[\text { SSE-192053(0,2)] }}\end{array}$ & $\begin{array}{l}(-2,0)(-2,2)(-2,4) \\
(0,0)(0,2)(0,4) \\
(2,0)(2,2)(2,4) \\
{[\text { SSE-100793(-2,4)] }}\end{array}$ & $\begin{array}{l}(-4,2)(-4,4)(-4,6) \\
(-2,2)(-2,4)(-2,6) \\
(0,2)(0,4)(0,6) \\
{[\text { SSE-91208(-2,6)] }}\end{array}$ & $\begin{array}{l}(-3,5)(-3,6) \\
(-3,7)(-2,5) \\
(-2,6)(-2,7) \\
(-1,5)(-1,6) \\
(-1,7)[\text { SSE-89338 }(-2,7)]\end{array}$ \\
\hline 3 & DS & $\begin{array}{l}(0,0)(-2,0)(-1,-1) \\
(-1,1)(0,-2)(0,2) \\
(1,-1)(1,1)(2,0) \\
{[\text { SSE-192053(0,2)] }}\end{array}$ & $\begin{array}{l}(0,2)(-1,1)(-1,3) \\
(0,0)(0,4)(1,1) \\
(1,1)(2,2)(-2,2) \\
{[\text { SSE-106219(0,4)] }}\end{array}$ & $\begin{array}{l}(0,4)(-1,3)(-1,5) \\
(0,2)(0,6)(1,3) \\
(1,5)(2,4)(-2,4) \\
\text { [SSE-97676(-1,5)] }\end{array}$ & $\begin{array}{l}(-2,4)(-2,5) \\
(-2,6)(-1,4) \\
(-1,5)(-1,6) \\
(0,4)(0,5) \\
(0,6)[\text { SSE-91208] }\end{array}$ \\
\hline 4 & $\begin{array}{l}\text { PBSMC } \\
\text { (PBSMCT) }\end{array}$ & $\begin{array}{l}(0,0)(0,-1)(0,-2)(0,1) \\
(0,2)(-1,0)(-1,-1)(-1,1) \\
(-2,0)(1,0)(1,-1)(1,1) \\
(2,0)(-6,0)(6,0)(-3,-3) \\
(-3,3)(3,-3)(3,3)(0,-6) \\
(0,6)(-6,0)(-3,-3) \\
(-3.3)(0,-6)(3,-3)(3,3) \\
(6,0)[\text { SSE-55970 }(0,-6)]\end{array}$ & $\begin{array}{l}(0,-6)(-2,-6)(-1,-7) \\
(-1,-5)(1,-7)(1,-5) \\
(2,-6)(0,-4) \\
{[\text { SSE-5890(1,-5)] }}\end{array}$ & $\begin{array}{l}(1,-5)(-1,-5)(0,-6)(0,- \\
4) \\
(1,-7)(1,-3)(2,-6)(2,- \\
4) \\
(3,-5)[\text { SSE-5702(3,- } \\
5)\end{array}$ & $\begin{array}{l}(3,-5)(1,-5) \\
(2,-6)(2,-4) \\
(3,-7)(3,-3) \\
(4,-6)(4,-4) \\
(5,-5)[\text { SSE-5271 }(5,-5)]\end{array}$ \\
\hline
\end{tabular}

TABLE. X COMPARISON OF THE AVERAGE PSNR (DB) VALUES OF FS, PBSMC, PBSMCT, DS, FSS AND TSS

\begin{tabular}{|c|c|c|c|c|c|c|}
\hline Sequence & FS & PBSMC & PBSMCT & DS & FSS & TSS \\
\hline Miss America & 41.866 & 41.878 & 41.84 & 41.78 & 41.618 & 41.587 \\
\hline Suzie & 37.422 & 37.431 & 37.428 & 37.157 & 37.006 & 36.89 \\
\hline Akiwo & 44.776 & 44.772 & 44.772 & 44.763 & 44.665 & 44.647 \\
\hline Carphone & 33.654 & 33.672 & 33.668 & 33.106 & 32.971 & 32.99 \\
\hline Coastguard & 33.804 & 33.8 & 33.8 & 33.688 & 33.59 & 33.173 \\
\hline Container & 43.271 & 43.274 & 43.269 & 43.187 & 43.202 & 43.226 \\
\hline Foreman & 33.919 & 33.751 & 33.748 & 33.068 & 32.998 & 32.989 \\
\hline Hall Monitor & 37.698 & 37.695 & 37.693 & 37.666 & 37.622 & 37.589 \\
\hline Mobile & 26.332 & 26.328 & 26.328 & 26.302 & 26.304 & 26.296 \\
\hline Mthr \& & 42.185 & 42.217 & 42.201 & 42.083 & 42.022 & 41.807 \\
\hline News & 37.923 & 37.878 & 37.877 & 37.652 & 37.571 & 37.451 \\
\hline Silent & 37.555 & 37.594 & 37.594 & 37.17 & 37.094 & 37.028 \\
\hline Salesman & 41.248 & 41.244 & 41.241 & 41.192 & 41.13 & 41.064 \\
\hline Claire & 43.362 & 43.355 & 43.34 & 43.342 & 43.307 & 43.27 \\
\hline Grandma & 43.673 & 43.677 & 43.647 & 43.645 & 43.632 & 43.632 \\
\hline Highway & 36.376 & 35.891 & 35.89 & 35.579 & 35.398 & 35.385 \\
\hline Bridge (Close) & 38.576 & 38.576 & 38.575 & 38.575 & 38.575 & 38.574 \\
\hline Bridge(Far) & 41.648 & 41.641 & 41.64 & 41.616 & 41.611 & 41.611 \\
\hline Stefan & 27.32 & 26.647 & 26.647 & 25.055 & 25.128 & 25.907 \\
\hline Bus & 26.808 & 25.316 & 25.316 & 22.85 & 22.758 & 24.062 \\
\hline Flower & 27.204 & 27.116 & 27.115 & 26.909 & 26.53 & 24.038 \\
\hline Tempete & 27.926 & 28.213 & 28.213 & 27.709 & 27.661 & 27.694 \\
\hline Waterfall & 34.794 & 34.794 & 34.794 & 34.792 & 34.791 & 34.767 \\
\hline Paris & 35.174 & 35.119 & 35.119 & 34.79 & 34.686 & 34.625 \\
\hline
\end{tabular}

TABLE. XI COMPARISON OF AVERAGE NumBER OF SEARCH POINTS OF FS, PBSMC, PBSMCT, DS, FSS AND TSS

\begin{tabular}{|l|c|c|c|c|c|c|}
\hline \multicolumn{1}{|c|}{ Sequence } & FS & PBSMC & PBSMCT & DS & FSS & TSS \\
\hline Miss America & 204.28 & 22.47 & 6.88 & 13.69 & 16.756 & 23.268 \\
\hline Suzie & 204.28 & 22.11 & 14.51 & 13.49 & 16.579 & 23.256 \\
\hline Akiyo & 204.28 & 10.56 & 4.31 & 12.22 & 15.816 & 23.212 \\
\hline Carphone & 204.28 & 23.81 & 17.31 & 14.71 & 17.131 & 23.322 \\
\hline Coastguard & 204.28 & 22.48 & 20.44 & 14.16 & 17.03 & 23.286 \\
\hline Container & 204.28 & 20.76 & 4.83 & 12.33 & 15.883 & 23.218 \\
\hline Foreman & 204.28 & 24.79 & 20.32 & 15.7 & 17.71 & 23.316 \\
\hline Hall Monitor & 204.28 & 21.11 & 16.85 & 12.65 & 16.118 & 23.254 \\
\hline Mobile & 204.28 & 20.96 & 20.55 & 12.33 & 15.869 & 23.216 \\
\hline Mthr \& & 204.28 & 21.78 & 8.08 & 13.15 & 16.493 & 23.261 \\
\hline News & 204.28 & 15.38 & 6.01 & 12.57 & 16.017 & 23.212 \\
\hline Silent & 204.28 & 21.52 & 8.6 & 12.94 & 16.227 & 23.218 \\
\hline Salesman & 204.28 & 20.73 & 6.4 & 12.29 & 15.858 & 23.214 \\
\hline Claire & 204.28 & 19.58 & 4.78 & 12.42 & 15.926 & 23.231 \\
\hline Grandma & 204.28 & 21.42 & 5.87 & 12.84 & 16.276 & 23.266 \\
\hline Highway & 204.28 & 21.7 & 20.43 & 13.72 & 16.801 & 23.336 \\
\hline Bridge(Close) & 204.28 & 18.59 & 17.57 & 12.22 & 15.829 & 23.225 \\
\hline Bridge(Far) & 204.28 & 21.16 & 19.91 & 12.65 & 16.048 & 23.218 \\
\hline Stefan & 214.52 & 27.46 & 22.34 & 17.11 & 18.733 & 24.141 \\
\hline Bus & 214.52 & 31.75 & 31.5 & 20.08 & 20.388 & 24.312 \\
\hline Flower & 214.52 & 25.7 & 18.07 & 16.7 & 19.172 & 24.157 \\
\hline Tempete & 214.52 & 23.55 & 20.28 & 14.27 & 17.202 & 24.141 \\
\hline Waterfall & 214.52 & 21.58 & 20.49 & 12.7 & 16.431 & 24.099 \\
\hline Paris & 214.52 & 21.71 & 8.92 & 12.95 & 16.605 & 24.099 \\
\hline
\end{tabular}

\section{CONCLUSION}

Our proposed algorithm, PBSMC, has been presented in this paper for motion estimation. The proposed algorithm uses stochastic approach to locate the true motion vector. Simulation results show that PBSMC achieves better estimate accuracy as compared to earlier proposed algorithms. Due to its efficient search pattern it track motion vector in vicinity of central point as well as at edge of the search window which makes it more applicable search algorithm for video with small and large motion. Mean correction technique developed, considerably improves PSNR at much reduced computational complexity. The early termination of algorithm with reasonable PSNR is possible using PBSMCT algorithm, using the threshold and mean correction technique. The computations involved in this technique are considerably 
low as compared to FS, TSS, FSS, DS, and PBSMC with PSNR comparable to PBSMC and much better than other sub optimal algorithm. In addition proposed algorithm is more robust as compared to earlier algorithms because it is flexible enough to work well, for any search range and window size which will be useful in rate constrained environment. Even the performance of PBSMC is consistent for the image sequence that contains complex movements such as camera panning and zooming. The simulation result demonstrates that the proposed algorithm is very suitable for high quality video encoding.

\section{REFERENCES}

[1] B. Haskell, A. Puri, A.Netravali, "Digital video: An introduction to MPEG-2", Kluwer Academic Publisher, 2000.

[2] K.Jack, "Video demystified: A Handbook for the Digital Engineer", Elsevier Publisher, Linacre House, Oxford, 2005

[3] T.Koga,K.Iinuma,A.Hirano,Y.Iijima, and T.Ishiguro,"Motion compensated interframe coding for video conferencing," in Proc. National Telecommunications Conf., Nov. 29-Dec. 3, 1981, pp G.5.3.1-G.5.3.5

[4] Li and E Salari, "Successive Elimination Algorithm for Motion Estimation", IEEE Transactions on Image Processing, vol. 4, no. 1, pp. 105-107, Jan.1995.

[5] B.Zeng, and M.Liou, "A New Three Step Search Algorithm for Block Motion Estimation," IEEE Trans. Circuits Syst. Video Technol., vol. 4, no.4, pp. 438-442, Aug. 1994.

[6] Lai-Man Po and Wing-Chung Ma, "A Novel Four-Step Search Algorithm for Fast Block Motion Estimation", IEEE Transactions on Circuits and Systems for Video Technology, vol. 6, no. 3, pp. 313317 , June 1996.

[7] Kuan-Tsang wang, "Efficient Four-Step Search", IEEE international symposium on Circuits and Systems, 1998, ISCAS apos,'98 vol. 4, pp. 217-220, June 1998.

[8] Jo Yew Tham, Surendra Ranganath, Maitreya Ranganath and Ashraf Ali Kassim, "A Novel Unrestricted Centre Biased Diamond Search Algorithm for Motion Estimation", IEEE Transactions on Circuits and Systems for Video Technology, vol. 8, no. 4, pp. 369-377, Aug. 1998.

[9] M.Ghanbari, "The cross search algorithm for motion estimation," IEEE Trans.commun.,vol.38, no.7, pp.950-953, July 1990.

[10] Jong-Nam Kim, Sung-Cheal Byun, Yong-Hoon Kim, and Byung-Ha Ahn, "Fast Full Search Motion Estimation Algorithm using Early Detection of Impossible Candidate Vectors", IEEE Transaction on Signal Processing, vol. 50, no. 9, pp.2355-2365, Sept. 2002.

[11] Antonio Chimienti, Claudia Ferraris, and Danilo Pau, "A complexity-bounded motion estimation algorithm", IEEE Transactions on image processing, vol. 11, no. 4, pp.387-392, Apr. 2002.

[12] B.Liu and A. Zaccartin, "New Fast Algorithms for Estimation of Block Motion Vectors," IEEE Trans. Circuits Syst. Video Technol., vol. 3, no.2, pp.148-157, Apr.1993.

[13] L.W.Lee, J.F. Wang, J.Y.Lee, and J.D. Shie, “ Dynamic Search Window Adjustment and Interlaced Search for Block-Matching Algorithm, " IEEE Trans. Circuits Syst. Video Technol., vol.3, no.1, pp.85-87, Feb.1999

[14] R. Srinivasan and K.R. Rao, "Predictive Coding Based on Efficient Motion Estimation," IEEE Trans. Circuits Syst.Video Technol., vol.Comm-33, no.8, pp.888-896, Aug.1985.

[15] J. R. Jain and A. K. Jain, "Displacement measurement and its application in interframe image coding," IEEE Trans. Commun., vol. COM-29, no.12, pp.1799-1808, Dec.1981.

[16] Hanan, suneer, mohsen and magdy bayoumi, "A new efficient block-matching algorithm for motion estimation" Journal of VLSI Signal processing system, vol. 42, issue.1, pp.21-33, Jan. 2006.

[17] Z. Zhou, M.T. Sun, S. Hsu, "Fast Variable Block-size Motion Estimation Algorithms Based on Merge and Split Procedure for H.264/MPEG-4 AVC," IEEE ISCAS Conference. 2004.
[18] Ishfaq Ahmad, Weiguo Zheng, Jiancong Luo and Ming Liou "A Fast Adaptive Motion Estimation Algorithm" IEEE Trans. Circuits Syst. Video Technol., vol.16, no.3, pp.420-438, Mar.2006.

[19] A. M. Tourapis, O. C. Au, and M. L. Liou, "Fast block-matching motion estimation using advanced predictive diamond zonal search (APDZS), ISO/IEC JTC1/SC29/WG11 MPEG2000/M5865, Noordwijkerhout, The Netherlands, Mar., 2000.

[20] "Fast block-matching motion estimation using predictive motion vector field adaptive search technique (PMVFAST),"ISO/IECJTC1/SC29/WG11 MPEG2000/M5866, Noordwijkerhout, The Netherlands, Mar. 2000.

[21] J. Feng, K. T. Lo, H. Mehrpour, and A. E. Karbowiak, "Adaptive blockmatching motion estimation algorithm for video coding," IEE Electron. Lett., vol. 31, no.18, pp. 1542-1543, 1995.

[22] B. C. Song and J. B. Ra, "A hierarchical block matching algorithm using partial distortion criteria," in Proc. VCIP Vis. Commun. Image Process.,San Jose, CA, pp.88-95. 1998.

[23] J. B. Xu, L. M. Po, and C. K. Cheung, "A new prediction model search algorithm for fast block motion estimation," in IEEE Int. Conf. ImageProcess., 1997, pp. 610-613. 1997.

[24] T. Wiegand, G. J. Sullivan, G. Bjontegaard, and A. Luthra, "Overview of the H.264/AVC Video Coding Standard," IEEE Transactions on Circuits and Systems for Video Technology, vol.13, no.7, pp. 560576, 2003.

[25] Kuo-Liang chung and Lung-chung chang, " A New Predictive Search Area Approach for Fast Block Motion Estimation," IEEE Transaction on Image Processing, vol.12, no.6, pp. 648-652, June 2003.

[26] Ce-Zhu, Wei-Song Qi, and Ser-W, "Predictive Fine Granularity Successive Elimimination for Fast Optimal Block- Matching motion Estimation," IEEE Transaction on Image Processing, vol.14, no.2, pp.213-221 Feb.2005.

[27] Shen-Chen Tai, Ying-Ru Chen, Yu-Hung chen, "Small-diamond-based search algorithm for fast block motion estimation," Elsevier Publisher, Signal Processing: Image Communication, vol. 22, no.10, pp. 877- 890, Nov. 2007

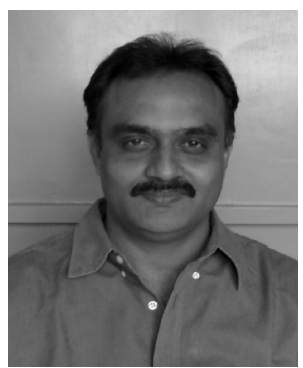

Deepak J.Jayaswal received his B.E degree in electronics engineering from Walchand College of engineering, Shivaji University, Kolhapur, Maharashtra, India in 1991, M-Tech degree in communication from IIT Bombay, 2002. He is student member IEEE. He is working in St. Francis Institute of Technology, Borivali, Mumbai as Assistant Professor in Electronics and Telecommunication department, His research area includes Image and video processing.

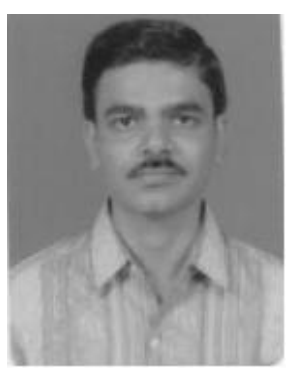

Mukesh A. Zaveri received the B.E. degree in electronics engineering from Sardar Vallabhbhai Regional College of Engineering and Technology, Surat, India, in 1990, the M.E. degree in electrical engineering from Maharaja Sayajirao University, Baroda, India, in 1993, and the Ph.D. degree in electrical engineering from the Indian Institute of Technology Bombay, in 2005. He is currently an Assistant Professor and the Head of the Computer Engineering Department, Sardar Vallabhbhai National Institute of Technology. His current research interests include the area of signal and image processing, multimedia, computer networks, sensor networks, and wireless communications. 